\title{
Construction on the Degenerate Poly-Frobenius-Euler Polynomials of Complex Variable
}

\author{
Ghulam Muhiuddin $\mathbb{D}^{1}{ }^{1}$ Waseem Ahmad Khan, ${ }^{2}$ and Deena Al-Kadi ${ }^{3}$ \\ ${ }^{1}$ Department of Mathematics, University of Tabuk, Tabuk 71491, Saudi Arabia \\ ${ }^{2}$ Department of Mathematics and Natural Sciences, Prince Mohammad Bin Fahd University, P.O Box 1664, \\ Al Khobar 31952, Saudi Arabia \\ ${ }^{3}$ Department of Mathematics and Statistics, College of Science, Taif University, P.O. Box 11099, Taif 21944, Saudi Arabia
}

Correspondence should be addressed to Ghulam Muhiuddin; chishtygm@gmail.com

Received 12 June 2021; Accepted 2 August 2021; Published 21 August 2021

Academic Editor: Sarfraz Nawaz Malik

Copyright (c) 2021 Ghulam Muhiuddin et al. This is an open access article distributed under the Creative Commons Attribution License, which permits unrestricted use, distribution, and reproduction in any medium, provided the original work is properly cited.

\begin{abstract}
In this paper, we introduce degenerate poly-Frobenius-Euler polynomials and derive some identities of these polynomials. We give some relationships between degenerate poly-Frobenius-Euler polynomials and degenerate Whitney numbers and Stirling numbers of the first kind. Moreover, we define degenerate poly-Frobenius-Euler polynomials of complex variables and then we derive several properties and relations.
\end{abstract}

\section{Introduction}

Recently, many mathematicians, namely, Carlitz [1,2], Kim and Kim [3-5], Kim et al. [6-9], Muhiuddin et al. [10-12], and Sharma et al. [13-15] have introduced and studied various degenerate versions of special polynomials and numbers like degenerate Bernoulli polynomials, degenerate Euler polynomials, degenerate Daehee polynomials, degenerate Fubini polynomials, and degenerate Stirling numbers of the first and second kinds.

The classical Frobenius-Euler polynomials $\mathbb{H}_{n}^{(\alpha)}(x ; u)$ $(u \in \mathbb{C}$ with $u \neq 1$ ) of order $\alpha$ are defined by means of the following generating function (see $[16,17])$ :

$$
\left(\frac{1-u}{e^{z}-u}\right)^{\alpha} e^{\zeta z}=\sum_{j=0}^{\infty} \mathbb{H}_{j}^{(\alpha)}(\zeta ; u) \frac{z^{j}}{j !}
$$

At the point $\zeta=0, \mathbb{H}_{j}^{(\alpha)}(u)=\mathbb{H}_{j}^{(\alpha)}(0 ; u)$ are called $j^{\text {th }}$ Frobenius-Euler numbers of order $\alpha$.
The poly-Frobenius-Euler polynomials due to Kurt [16] are defined as follows:

$$
\frac{(1-u) L i_{k}\left(1-e^{-z}\right)}{z\left(e^{z}-u\right)} e^{\zeta z}=\sum_{j=0}^{\infty} \mathbb{H}_{j}^{(k)}(\zeta ; u) \frac{z^{j}}{j !} .
$$

When $\zeta=0, \mathbb{H}_{j}^{(k)}(u)=\mathbb{H}_{j}^{(k)}(0 ; u)$ are called the polyFrobenius-Euler numbers.

For any $\lambda \in \mathbb{R}$ (or $\mathbb{C}$ ), $\mathbb{R}$ and $\mathbb{C}$ being, respectively, the sets of real numbers and complex numbers, degenerate version of the exponential function $e_{\lambda}^{\zeta}(z)$ is defined as follows (see $[3,4,6,18])$ :

$$
e_{\lambda}^{\zeta}(z):=(1+\lambda z)^{\zeta / \lambda}=\sum_{j=0}^{\infty}(\zeta)_{j, \lambda} \frac{z^{j}}{j !}
$$

where $(\zeta)_{0, \lambda}=1$ and $(\zeta)_{j, \lambda}=\zeta(\zeta-\lambda) \cdots(\zeta-(j-1) \lambda)$ for $j \geq 1$ (see $[1,2,4-10,18]$ ). It follows from Equation (3) that $\lim _{\lambda \longrightarrow 0} e_{\lambda}^{\zeta}(z)=e^{\zeta z}$. Note that $e_{\lambda}^{1}(z):=e_{\lambda}(z)$. 
Carlitz [1, 2] introduced the degenerate Euler polynomials as follows:

$$
\frac{2}{e_{\lambda}(z)+1} e_{\lambda}^{x}(z)=\sum_{j=0}^{\infty} E_{j, \lambda}(x) \frac{z^{j}}{j !}
$$

In the case when $\zeta=0, E_{j, \lambda}=E_{j, \lambda}(0)$ are called the degenerate Euler numbers.

Note that

$$
\lim _{\lambda \longrightarrow 0} E_{j}(\zeta ; \lambda)=E_{j}(\zeta)
$$

The degenerate Frobenius-Euler polynomials are defined by the following (see [6]):

$$
\frac{1-u}{(1+\lambda z)^{1 / \lambda}-u}(1+\lambda z)^{\zeta / \lambda}=\sum_{j=0}^{\infty} h_{j, \lambda}(\zeta \mid u) \frac{z^{j}}{j !} .
$$

At the value $\zeta=0, h_{j, \lambda}(u)=h_{j, \lambda}(0 \mid u)$ are called the degenerate Frobenius-Euler numbers.

It is readily seen that

$$
\lim _{\lambda \longrightarrow 0} h_{j, \lambda}(\zeta \mid u)=\mathbb{H}_{j}(\zeta \mid u), \quad(j \geq 0)
$$

Recently, Kim et al. [9] introduced the modified degenerate polyexponential function which is defined by the following:

$$
E i_{k, \lambda}(\zeta)=\sum_{j=1}^{\infty} \frac{(1)_{j, \lambda} \zeta^{j}}{(j-1) ! j^{k}}, \quad(|\zeta|<1, k \in \mathbb{Z})
$$

Here and in the following, let $\mathbb{Z}$ denote the set of integers. We note that

$$
E i_{1, \lambda}(\zeta)=\sum_{j=1}^{\infty} \frac{(1)_{j, \lambda} \zeta^{j}}{j !}=e_{\lambda}(\zeta)-1
$$

The degenerate poly-Genocchi polynomials are defined as follows (see [9]):

$$
\frac{2 E i_{k, \lambda}\left(\log _{\lambda}(1+z)\right)}{e_{\lambda}(z)+1} e_{\lambda}^{\zeta}(z)=\sum_{j=0}^{\infty} G_{j, \lambda}^{(k)}(\zeta) \frac{z^{j}}{j !}, \quad(k \in \mathbb{Z})
$$

Letting $\zeta=0, G_{j, \lambda}^{(k)}=G_{j, \lambda}^{(k)}(0)$ are called the poly-Genocchi numbers.

The degenerate Daehee polynomials $D_{j, \lambda}(\zeta)$ are defined as follows (see [8]):

$$
\frac{\log _{\lambda}(1+z)}{z}(1+z)^{\zeta}=\sum_{j=0}^{\infty} D_{j, \lambda}(\zeta) \frac{z^{j}}{j !}
$$

$D_{j, \lambda}=D_{j, \lambda}(0)$ are called the degenerate Daehee numbers.

For $i \geq 0$, the degenerate Stirling numbers of the first kind are defined by means of the following generating function (see [4]):

$$
\frac{1}{i !}\left(\log _{\lambda}(1+z)\right)^{i}=\sum_{j=i}^{\infty} S_{1, \lambda}(j, i) \frac{z^{j}}{j !} .
$$

Note that $\lim _{\lambda \longrightarrow 0} S_{1, \lambda}(j, k)=S_{1}(j, k)$ are the Stirling numbers of the first kind given by the following (see $[3,18])$ :

$$
\frac{1}{i !}(\log (1+z))^{i}=\sum_{j=i}^{\infty} S_{1}(j, i) \frac{z^{j}}{j !}, \quad(i \geq 0)
$$

For $i \geq 0$, the degenerate Stirling numbers of the second kind are defined by means of the following generating function (see [18]):

$$
\frac{1}{i !}\left(e_{\lambda}(z)-1\right)^{i}=\sum_{j=i}^{\infty} S_{2, \lambda}(j, i) \frac{z^{j}}{j !} .
$$

We note that $\lim _{\lambda \longrightarrow 0} S_{2, \lambda}(j, k)=S_{1}(j, k)$ are the Stirling numbers of the second kind given by the following (see $[3-7,18])$

$$
\frac{1}{i !}\left(e^{z}-1\right)^{i}=\sum_{j=i}^{\infty} S_{2}(j, i) \frac{z^{j}}{j !}, \quad(i \geq 0) .
$$

The subsequent content of this paper is organized as follows: In Section 2, we define the degenerate polyFrobenius-Euler polynomials and numbers by using the modified degenerate polyexponential functions and derive some properties and relations of these polynomials. In Section 3, we consider the degenerate poly-Frobenius-Euler polynomials of a complex variable and then we derive several properties and relations. Also, we examine the results derived in this study.

\section{Degenerate Poly-Frobenius-Euler Numbers and Polynomials}

In this section, we define degenerate poly-Frobenius-Euler numbers and polynomials and investigate some properties of these polynomials. We begin following the definition as follows. 
Definition 1. We consider the degenerate poly-FrobeniusEuler polynomials are defined by means of the following generating function:

$$
\frac{E i_{k, \lambda}\left(\log _{\lambda}(1+(1-u)) z\right)}{z\left(e_{\lambda}(z)-u\right)} e_{\lambda}^{\zeta}(z)=\sum_{j=0}^{\infty} \mathbb{H}_{j, \lambda}^{(k)}(\zeta ; u) \frac{z^{j}}{j !}
$$

where $\lambda, u \in \mathbb{C}$ with $u \neq 1$ and $k \in \mathbb{Z}$.

Upon setting, $\zeta=0, \mathbb{H}_{j, \lambda}^{(k)}(u)=\mathbb{H}_{j, \lambda}^{(k)}(0 ; u)$ are called the degenerate poly-Frobenius-Euler numbers, where $\log _{\lambda}(z)=$ $1 / z\left(z^{\lambda}-1\right)$ is the compositional inverse of $e_{\lambda}(z)$ satisfying

$$
\log _{\lambda}\left(e_{\lambda}(z)\right)=e_{\lambda}\left(\log _{\lambda}(z)\right)=z
$$

Adjusting $k=1$ in Equation (16), we get the following:

$$
\frac{1-u}{e_{\lambda}(z)-u} e_{\lambda}^{\zeta}(z)=\sum_{j=0}^{\infty} h_{j, \lambda}(\zeta ; u) \frac{z^{j}}{j !}
$$

where $h_{j, \lambda}(\zeta ; u)$ are called the degenerate Frobenius-Euler polynomials (see [6]).

Obviously,

$$
\begin{aligned}
\lim _{\lambda \longrightarrow 0}\left(\frac{E i_{k, \lambda}\left(\log _{\lambda}(1+(1-u) z)\right)}{z\left(e_{\lambda}(z)-u\right)}\right) e_{\lambda}^{\zeta}(z)=\sum_{j=0}^{\infty} \lim _{\lambda \longrightarrow 0} \mathbb{H}_{j, \lambda}^{(k)}(\zeta ; u) \frac{z^{j}}{j !} \\
\quad=\frac{E i_{k}(\log (1+(1-u) z))}{z\left(e^{z}-u\right)} e^{\zeta z} \\
\quad=\sum_{j=0}^{\infty} \mathbb{H}_{j}^{(k)}(\zeta ; u) \frac{z^{j}}{j !},
\end{aligned}
$$

where $\mathbb{H}_{j}^{(k)}(\zeta ; u)$ are called the type 2 poly-Frobenius-Euler polynomials.

Theorem 2. Let $j \geq 0$. Then, we have the following:

$$
\begin{aligned}
& \sum_{p=0}^{j}\left(\begin{array}{l}
j \\
p
\end{array}\right) \sum_{s=0}^{p} \frac{(1)_{s+1, \lambda}}{(s+1)^{k-1}} S_{1, \lambda}(p+1, s+1)(\zeta)_{j-p, \lambda} \frac{(1-u)^{p+1}}{p+1} \\
& \quad=\sum_{s=0}^{j}\left(\begin{array}{l}
j \\
s
\end{array}\right) \mathbb{H}_{j-s, \lambda}^{(k)}(\zeta ; u)(1)_{s, \lambda}-u \mathbb{H}_{j, \lambda}^{(k)}(\zeta ; u) .
\end{aligned}
$$

Proof. Using Equation (16), we see that

$$
\begin{aligned}
& \frac{E i_{k, \lambda}\left(\log _{\lambda}(1+(1-u) z)\right)}{z} e_{\lambda}^{\zeta}(z) \\
& \quad=e_{\lambda}(z) \sum_{j=0}^{\infty} \mathbb{H}_{j, \lambda}^{(k)}(\zeta ; u) \frac{z^{j}}{j !}-u \sum_{j=0}^{\infty} \mathbb{H}_{j, \lambda}^{(k)}(\zeta ; u) \frac{z^{j}}{j !} \\
& \quad=\sum_{s=0}^{\infty}(1)_{s, \lambda} \frac{z^{s}}{s !} \sum_{j=0}^{\infty} \mathbb{H}_{j, \lambda}^{(k)}(\zeta ; u) \frac{z^{j}}{j !}-u \sum_{j=0}^{\infty} \mathbb{H}_{j, \lambda}^{(k)}(\zeta ; u) \frac{z^{j}}{j !} \\
& =\sum_{j=0}^{\infty}\left(\sum_{s=0}^{j}\left(\begin{array}{l}
j \\
s
\end{array}\right) \mathbb{H}_{j-s, \lambda}^{(k)}(\zeta ; u)(1)_{s, \lambda}-u \mathbb{H}_{j, \lambda}^{(k)}(\zeta ; u)\right) \frac{z^{j}}{j !} .
\end{aligned}
$$

On the other hand,

$$
\begin{aligned}
& \frac{E i_{k, \lambda}\left(\log _{\lambda}(1+(1-u) z)\right)}{z} e_{\lambda}^{\zeta}(z) \\
& =\left(\sum_{j=0}^{\infty}(\zeta)_{j, \lambda} z^{j}\right) \frac{1}{z !}\left(\sum_{s=1}^{\infty} \frac{(1)_{s, \lambda}\left(\log _{\lambda}(1+(1-u) z)\right)^{s}}{(s-1) ! s^{k}}\right) \\
& =\left(\sum_{j=0}^{\infty}(\zeta)_{j, \lambda} z^{j}\right) \frac{1}{j !}\left(\sum_{s=0}^{\infty} \frac{(1)_{s+1, \lambda}}{(s+1)^{k-1}} \sum_{l=s+1}^{\infty} S_{1, \lambda}(l, s+1) \frac{(1-u)^{l} z^{l}}{l !}\right) \\
& =\left(\sum_{j=0}^{\infty}(\zeta)_{j, \lambda} z^{j}\right)\left(\sum_{l=0}^{\infty} \sum_{s=0}^{l} \frac{(1)_{s+1, \lambda}}{(s+1)^{k-1}} S_{1, \lambda}(l+1, s+1) \frac{(1-u)^{l+1} z^{l}}{l+1}\right) \\
& =\sum_{j=0}^{\infty}\left(\sum_{p=0}^{j}\left(\begin{array}{l}
j \\
p
\end{array}\right) \sum_{s=0}^{p} \frac{\left.(1)_{s+1, \lambda}(s+1)^{k-1} S_{1, \lambda}(p+1, s+1)(\zeta)_{j-p, \lambda} \frac{(1-u)^{p+1}}{p+1}\right) \frac{z^{j}}{j !} .}{(22)}\right.
\end{aligned}
$$

In view of Equation (22), we complete the proof.

Theorem 3. Let $j \geq 0$. Then, we have the following:

$$
\mathbb{H}_{j, \lambda}^{(k)}(\zeta ; u)=\sum_{r=0}^{j}\left(\begin{array}{l}
j \\
r
\end{array}\right) \mathbb{H}_{j-r, \lambda}^{(k)}(u)(\zeta)_{r, \lambda}
$$

Proof. In Equation (16), we observe that

$$
\begin{aligned}
\sum_{j=0}^{\infty} \mathbb{H}_{j, \lambda}^{(k)}(\zeta ; u) \frac{z^{j}}{j !} & =\left(\frac{E i_{k, \lambda}\left(\log _{\lambda}(1+(1-u) z)\right)}{z\left(e_{\lambda}(z)-u\right)}\right) e_{\lambda}^{\zeta}(z) \\
& =\sum_{j=0}^{\infty} \mathbb{H}_{j, \lambda}^{(k)}(u) \frac{z^{j}}{j !} \sum_{r=0}^{\infty}(\zeta)_{r, \lambda} \frac{z^{r}}{r !} \\
& =\sum_{j=0}^{\infty} \sum_{r=0}^{j}\left(\begin{array}{l}
j \\
r
\end{array}\right) \mathbb{H}_{j-r, \lambda}^{(k)}(u)(\zeta)_{r, \lambda} \frac{z^{j}}{j !}
\end{aligned}
$$

By Equations (16) and (24), we require at the desired result. 
Theorem 4. Let $j \geq 0$. Then,

$\mathbb{H}_{j, \lambda}^{(k)}(\zeta ; u)=\sum_{q=0}^{j}\left(\begin{array}{l}j \\ q\end{array}\right) \sum_{r=0}^{q} \frac{(1)_{r+1, \lambda}}{(r+1)^{k}} \frac{S_{1}(q+1, r+1)(1-u)^{q}}{q+1} \mathbb{H}_{j-q, \lambda}(\zeta ; u)$.

Proof. By using Equations (14) and (16), we see that

$$
\begin{aligned}
& \sum_{j=0}^{\infty} \mathbb{H}_{j, \lambda}^{(k)}(\zeta ; u) \frac{z^{j}}{j !}=\left(\frac{E i_{k, \lambda}\left(\log _{\lambda}(1+(1-u) z)\right)}{z\left(e_{\lambda}(z)-u\right)}\right) e_{\lambda}^{\zeta}(z) \\
& =\frac{e_{\lambda}^{\zeta}(z)}{z\left(e_{\lambda}(z)-u\right)} \sum_{r=1}^{\infty} \frac{(1)_{r, \lambda}\left(\log _{\lambda}(1+(1-u) z)\right)^{r}}{(r-1) ! r^{k}} \\
& =\frac{e_{\lambda}^{\zeta}(z)}{z\left(e_{\lambda}(z)-u\right)} \sum_{r=0}^{\infty} \frac{(1)_{r+1, \lambda}\left(\log _{\lambda}(1+(1-u) z)\right)^{r+1}}{r !(r+1)^{k}} \\
& =\frac{e_{\lambda}^{\zeta}(z)}{z\left(e_{\lambda}(z)-u\right)} \sum_{r=0}^{\infty} \frac{(1)_{r+1, \lambda}}{(r+1)^{k}} \sum_{j=r+1}^{\infty} S_{1, \lambda}(j, r+1) \frac{((1-u) t)^{n}}{n !} \\
& =\frac{1-u}{e_{\lambda}(z)-u} e_{\lambda}^{\zeta}(z) \sum_{r=0}^{\infty} \frac{(1)_{r+1, \lambda}}{(r+1)^{k}} \sum_{r=j}^{\infty} \frac{S_{1, \lambda}(j+1, r+1)(1-u)^{j}}{j+1} \frac{z^{j}}{j !} \\
& =\sum_{j=0}^{\infty} \mathbb{H}_{j, \lambda}(\zeta ; u) \frac{z^{j}}{j !} \sum_{l=0}^{\infty} \sum_{r=0}^{l} \frac{(1)_{r+1, \lambda}}{(r+1)^{k}} \frac{S_{1, \lambda}(l+1, r+1)(1-u)^{l}}{l+1} \frac{z^{l}}{l !} \\
& =\sum_{j=0}^{\infty}\left(\sum_{q=0}^{j}\left(\begin{array}{l}
j \\
q
\end{array}\right) \sum_{r=0}^{q} \frac{(1)_{r+1, \lambda}}{(r+1)^{k}} \frac{S_{1, \lambda}(q+1, r+1)(1-u)^{q}}{q+1} \mathbb{H}_{j-q, \lambda}(\zeta ; u)\right) \frac{z^{j}}{j !} .
\end{aligned}
$$

In view of Equation (26), we complete the proof.

Corollary 5. For $k \in \mathbb{Z}$ and $j \geq 0$. Then,

$$
\mathbb{H}_{j, \lambda}^{(k)}(u)=\sum_{q=0}^{j}\left(\begin{array}{l}
j \\
q
\end{array}\right) \sum_{r=0}^{q} \frac{1}{(r+1)^{k}} \frac{S_{1, \lambda}(q+1, r+1)(1-u)^{q}}{q+1} \mathbb{H}_{j-q, \lambda}(u) .
$$

Corollary 6. For $j \geq 0$. Then,

$$
\mathbb{H}_{j, \lambda}^{(k)}(\zeta ; u)=\sum_{q=0}^{j}\left(\begin{array}{l}
j \\
q
\end{array}\right) \sum_{r=0}^{q} \frac{S_{1, \lambda}(q+1, r+1)(1-u)^{q}}{q+1} \mathbb{H}_{j-q, \lambda}(\zeta ; u)
$$

Corollary 7. On setting $u=-1$ and $k=1$ and using Equation (4), Theorem 3 to get

$E_{j, \lambda}(x)=\sum_{q=0}^{j}\left(\begin{array}{l}j \\ l\end{array}\right) \sum_{r=0}^{q} \frac{S_{1, \lambda}(q+1, r+1) 2^{q}}{q+1} E_{j-q, \lambda}(\zeta), \quad(j \geq 0)$.

It is well known from [7] that

$$
\left(\frac{z}{\log (1+z)}\right)^{r}(1+z)^{\zeta-1}=\sum_{j=0}^{\infty} B_{j}^{(j-r+1)}(\zeta) \frac{z^{j}}{j !}, \quad(r \in \mathbb{C}),
$$

where $B_{j}^{(r)}(\zeta)$ are called the higher-order Bernoulli polynomials which are given by the generating function (see $[3,16])$ :

$$
\left(\frac{z}{e^{z}-1}\right)^{r} e^{\zeta z}=\sum_{j=0}^{\infty} B_{j}^{(r)}(\zeta) \frac{z^{j}}{j !}
$$

Theorem 8. For $j \geq 0$. Then, we have the following:

$$
\mathbb{H}_{j, \lambda}^{(2)}(u)=\sum_{l=0}^{j}\left(\begin{array}{l}
j \\
l
\end{array}\right) \frac{(1-u)^{l} B_{l}^{l}}{l+1} \mathbb{H}_{j-l, \lambda}(u)
$$

Proof. In Equation (8), we note that

$$
\begin{aligned}
& \frac{d}{d \zeta} E i_{k, \lambda}\left(\log _{\lambda}(1+(1-u) x)\right) \\
& \quad=\frac{d}{d \zeta} \sum_{j=1}^{\infty} \frac{\left(\log _{\lambda}(1+(1-u) \zeta)\right)^{j}}{(j+1) ! j^{k}} \\
& \quad=\frac{1-u}{(1+(1-u) \zeta) \log _{\lambda}(1+(1-u) \zeta)} \sum_{j=1}^{\infty} \frac{\left(\log _{\lambda}(1+(1-u) \zeta)\right)^{j}}{(j+1) ! j^{k-1}} \\
& =\frac{1-u}{(1+(1-u) \zeta) \log _{\lambda}(1+(1-u) \zeta)} E i_{k-1, \lambda}\left(\log _{\lambda}(1+(1-u) \zeta)\right) .
\end{aligned}
$$

From Equation (33), for $k \geq 1$, we have the following:

$$
\begin{aligned}
& \sum_{j=0}^{\infty} \mathbb{H}_{j, \lambda}^{(k)}(u) \frac{\zeta^{j}}{j !}=\frac{(1-u)^{k-1}}{\zeta\left(e_{\lambda}(\zeta)-u\right)} \int_{0}^{\zeta} \frac{1}{(1+(1-u) t) \log _{\lambda}(1+(1-u) z)} \\
& \quad \times \int_{0}^{z} \underbrace{\frac{1}{(1+(1-u) z) \log _{\lambda}(1+(1-u) z)}}_{k-2 \text {-times }} \cdots \\
& \quad \cdot \int_{0}^{z} \frac{z}{(1+(1-u) z) \log _{\lambda}(1+(1-u) z)} d z d z \cdots d z .
\end{aligned}
$$

For $k \geq 2$ in the above expression, we have the following:

$$
\sum_{j=0}^{\infty} \mathbb{H}_{j, \lambda}^{(2)}(u) \frac{\zeta^{j}}{j !}=\frac{(1-u)}{\zeta\left(e_{\lambda}(\zeta)-u\right)} \int_{0}^{\zeta} \frac{(1-u) z}{(1+(1-u) z) \log _{\lambda}(1+(1-u) z)},
$$

$$
\left(\sum_{j=0}^{\infty} H_{j, \lambda}(u) \frac{\xi^{j}}{j !}\right)\left(\sum_{l=0}^{\infty} \frac{(1-u)^{l} B_{l}^{l}}{l+1} \frac{\xi^{l}}{l !}\right)=\sum_{j=0}^{\infty}\left(\sum_{l=0}^{j}\left(\begin{array}{l}
j \\
l
\end{array}\right) \frac{(1-u)^{l} B_{l}^{l}}{l+1} H_{j-l, \lambda}(u)\right) \frac{\xi^{j}}{j !}
$$

In view of Equations (35) and (36), we obtain at the desired result.

Theorem 9. Let $j \geq 0$. Then, we have the following:

$$
\mathbb{H}_{j, \lambda}^{(k)}(\zeta+\eta ; u)=\sum_{r=0}^{j}\left(\begin{array}{l}
j \\
r
\end{array}\right) \mathbb{H}_{j-r, \lambda}^{(k)}(\zeta ; u)(\eta)_{r, \lambda}
$$


Proof. Using Equation (16), we get the following:

$$
\begin{aligned}
\sum_{j=0}^{\infty} & \mathbb{H}_{j, \lambda}^{(k)}(\zeta+\eta ; u) \frac{z^{j}}{j !}=\left(\frac{\operatorname{Ei}_{k, \lambda}\left(\log _{\lambda}(1+(1-u) z)\right)}{z\left(e_{\lambda}(z)-u\right)}\right) e_{\lambda}^{\zeta+\eta}(z) \\
& =\left(\sum_{j=0}^{\infty} \mathbb{H}_{j, \lambda}^{(k)}(\zeta ; u) \frac{z^{j}}{j !}\right)\left(\sum_{r=0}^{\infty}(\eta)_{r, \lambda} \frac{z^{r}}{r !}\right) \\
& =\sum_{j=0}^{\infty}\left(\sum_{r=0}^{j}\left(\begin{array}{l}
j \\
r
\end{array}\right) \mathbb{H}_{j-r, \lambda}^{(k)}(\zeta ; u)(\eta)_{r, \lambda}\right) \frac{z^{j}}{j !} .
\end{aligned}
$$

Thus, by Equation (38), we complete the proof.

Theorem 10. Let $j \geq 0$. Then, we have the following:

$$
\mathbb{H}_{j, \lambda}^{(k)}(\zeta+1 ; u)=\sum_{r=0}^{j}\left(\begin{array}{l}
j \\
r
\end{array}\right) \mathbb{H}_{j-r, \lambda}^{(k)}(\zeta ; u)(1)_{r, \lambda}
$$

Proof. In Equation (16), we see that

$$
\begin{aligned}
& \sum_{j=0}^{\infty}\left[\mathbb{H}_{j, \lambda}^{(k)}(\zeta+1 ; u)-\mathbb{H}_{j, \lambda}^{(k)}(\zeta ; u)\right] \frac{z^{j}}{j !} \\
& \quad=\left(\frac{E i_{k, \lambda}\left(\log _{\lambda}(1+(1-u) z)\right)}{z\left(e_{\lambda}(z)-u\right)}\right) e_{\lambda}^{\zeta}(z)\left[e_{\lambda}(z)-1\right] \\
& \quad=\sum_{j=0}^{\infty} \sum_{r=0}^{j}\left(\begin{array}{c}
j \\
r
\end{array}\right) \mathbb{H}_{j-r, \lambda}^{(k)}(\zeta ; u)(1)_{r, \lambda} \frac{z^{j}}{j !}-\sum_{j=0}^{\infty} \mathbb{H}_{j, \lambda}^{(k)}(\zeta ; u) \frac{z^{j}}{j !} .
\end{aligned}
$$
result.

Comparing the coefficients of $z^{j}$ on both sides, we get the

Theorem 11. Let $j \geq 0$. Then,

$$
\mathbb{H}_{j, \lambda}^{(k)}(\zeta ; u)=\sum_{r=0}^{j} \sum_{q=0}^{r}\left(\begin{array}{l}
j \\
r
\end{array}\right)(\zeta)_{q} S_{2, \lambda}(r, q) \mathbb{H}_{j-r, \lambda}^{(k)}(u)
$$

Proof. From Equation (16), we see that

$$
\begin{aligned}
\sum_{j=0}^{\infty} & \mathbb{H}_{j, \lambda}^{(k)}(\zeta ; u) \frac{z^{j}}{j !}=\left(\frac{E i_{k, \lambda}\left(\log _{\lambda}(1+(1-u) z)\right)}{z\left(e_{\lambda}(z)-u\right)}\right) e_{\lambda}^{\zeta}(z) \\
& =\left(\frac{E i_{k, \lambda}\left(\log _{\lambda}(1+(1-u) z)\right)}{z\left(e_{\lambda}(z)-u\right)}\right)\left[e_{\lambda}(z)-1+1\right]^{\zeta} \\
& =\left(\frac{E i_{k, \lambda}\left(\log _{\lambda}(1+(1-u) z)\right)}{z\left(e_{\lambda}(z)-u\right)}\right)\left(\sum_{q=0}^{\infty}(\zeta)_{q} \sum_{l=q}^{\infty} S_{2, \lambda}(l, q) \frac{z^{l}}{l !}\right) \\
& =\sum_{j=0}^{\infty}\left(\sum_{r=0}^{j} \sum_{q=0}^{r}\left(\begin{array}{l}
j \\
r
\end{array}\right)(\zeta)_{q} S_{2, \lambda}(r, q) \mathbb{H}_{j-r, \lambda}^{(k)}(u)\right) \frac{z^{j}}{j !} .
\end{aligned}
$$

By Equation (42). We complete the proof.
Theorem 12. Let $j \geq 0$. Then,

$$
\mathbb{H}_{j, \lambda}^{(k)}(\zeta+\alpha \mid u)=\sum_{n=0}^{j} \sum_{l=0}^{n}\left(\begin{array}{l}
j \\
n
\end{array}\right) u^{l}(\zeta)_{l} W_{u, \alpha}(n, l ; \lambda) \mathbb{H}_{j-n, \lambda}^{(k)}(u)
$$

Proof. By changing $\zeta$ by $\zeta u+\alpha$ in Equation (16), we get the following:

$$
\begin{aligned}
\sum_{j=0}^{\infty} & \mathbb{H}_{j, \lambda}^{(k)}(\zeta+\alpha ; u) \frac{z^{j}}{j !}=\left(\frac{E i_{k, \lambda}\left(\log _{\lambda}(1+(1-u) z)\right)}{z\left(e_{\lambda}(z)-u\right)}\right) e_{\lambda}^{\alpha}(t) e_{\lambda}^{\zeta u}(z) \\
& =\left(\frac{E i_{k, \lambda}\left(\log _{\lambda}(1+(1-u) z)\right)}{z\left(e_{\lambda}(z)-u\right)}\right) e_{\lambda}^{\alpha}(t)\left[e_{\lambda}^{u}(z)-1+1\right)^{\zeta} \\
& =\left(\frac{E i_{k, \lambda}\left(\log _{\lambda}(1+(1-u) z)\right)}{z\left(e_{\lambda}(z)-u\right)}\right) e_{\lambda}^{\alpha}(z)\left(\sum_{l=0}^{\infty}\left(\begin{array}{c}
\zeta \\
l
\end{array}\right)\left[e_{\lambda}^{u}(z)-1\right)^{l}\right) \\
& =\left(\frac{E i_{k, \lambda}\left(\log _{\lambda}(1+(1-u) z)\right)}{z\left(e_{\lambda}(z)-u\right)}\right)\left(e_{\lambda}^{\alpha}(z) \sum_{l=0}^{\infty} u^{l}(\zeta)_{l} \operatorname{frac}\left(e_{\lambda}^{u}(z)-1\right)^{l} l ! u^{l}\right) \\
& =\sum_{j=0}^{\infty} \mathbb{H}_{j, \lambda}^{(k)}(u) \frac{z^{j}}{j !}\left(\sum_{n=0}^{\infty} \sum_{l=0}^{n} u^{l}(\zeta)_{l} W_{u, \alpha}(n, l ; \lambda) \frac{z^{n}}{n !}\right) \\
& =\sum_{j=0}^{\infty}\left(\sum_{n=0}^{j} \sum_{l=0}^{n}\left(\begin{array}{c}
j \\
n
\end{array}\right) u^{l}(\zeta)_{l} W_{u, \alpha}(n, l ; \lambda) \mathbb{H}_{j-n, \lambda}^{(k)}(u)\right) \frac{z^{j}}{j !} .
\end{aligned}
$$

Therefore, by Equations (16) and (44), we obtain the result.

\section{Degenerate Unipoly-Frobenius-Euler Numbers and Polynomials}

In this section, we introduce degenerate unipoly-FrobeniusEuler polynomials by using degenerate unipoly polynomials and derive some important properties of these polynomials.

In [3], Kim and Kim introduced unipoly function. In the view of [9], the degenerate unipoly function is defined by Dolgy and Khan [19] as follows:

$$
u_{k, \lambda}(\zeta \mid p)=\sum_{j=1}^{\infty} p(j) \frac{(1)_{j, \lambda} \zeta^{j}}{j^{k}}
$$

Note that, we have the following:

$$
u_{k, \lambda}\left(\zeta \mid \frac{1}{\Gamma}\right)=E i_{k, \lambda}(\zeta)
$$

is the modified degenerate polylogarithm function.

It is clear that

$$
\lim _{\lambda \longrightarrow 0} u_{k, \lambda}(\zeta \mid p)=\sum_{j=1}^{\infty} \lim _{\lambda \longrightarrow 0} p(i) \frac{(1)_{i, \lambda} \zeta^{i}}{i^{k}}=u_{k}(\zeta \mid p)=\sum_{j=1}^{\infty} p(i) \frac{\zeta^{i}}{i^{k}}
$$

are called the unipoly function attached to polynomials $p(\zeta)$ (see [20]). 
From Equation (47), we have the following:

$$
u_{k}(\zeta \mid 1)=\sum_{j=1}^{\infty} \frac{\zeta^{j}}{j^{k}}=L i_{k}(\zeta)(\operatorname{see}[9])
$$

is the ordinary polylogarithm function.

By using Equations (45) and (16), we define the degenerate unipoly-Frobenius-Euler polynomials as follows:

$$
\frac{u_{k, \lambda}\left(\log _{\lambda}(1+(1-u) z) \mid p\right)}{z\left(e_{\lambda}(z)-u\right)} e_{\lambda}^{\zeta}(z)=\sum_{j=0}^{\infty} \mathbb{H}_{j, \lambda, p}^{(k)}(\zeta ; u) \frac{z^{j}}{j !}
$$

At the special value $\zeta=0, \mathbb{H}_{j, \lambda, p}^{(k)}(u)=\mathbb{H}_{j, \lambda, p}^{(k)}(0 ; u)$ are called the degenerate unipoly-Frobenius-Euler numbers.

Theorem 13. Let $j \geq 0$. Then, we have the following:

$$
\mathbb{H}_{j, \lambda, 1 / \Gamma}^{(k)}(\zeta ; \eta)=\mathbb{H}_{j, \lambda}^{(k)}(\zeta ; u), \quad(k \in \mathbb{Z}) .
$$

Proof. Let us take $p(j)=1 / \Gamma \lambda$. Then, we have the following:

$$
\begin{aligned}
\sum_{j=0}^{\infty} & \mathbb{H}_{j, \lambda, 1 / \Gamma}^{(k)}(\zeta ; u) \frac{z^{j}}{j !}=\frac{u_{k, \lambda}\left(\log _{\lambda}(1+(1-u) z) \mid 1 / \Gamma p\right)}{z\left(e_{\lambda}(z)-u\right)} e_{\lambda}^{\zeta}(z) \\
& =\frac{1}{z\left(e_{\lambda}(z)-u\right)} \sum_{r=1}^{\infty} \frac{(1)_{r, \lambda}\left(\log _{\lambda}(1+(1-u) z)\right)^{r}}{r^{k}(r+1) !} e_{\lambda}^{\zeta}(z) \\
& =\frac{E i_{k, \lambda}\left(\log _{\lambda}(1+(1-u) z)\right)}{z\left(e_{\lambda}(z)-u\right)} e_{\lambda}^{\zeta}(z) \\
& =\sum_{j=0}^{\infty} \mathbb{H}_{j, \lambda}^{(k)}(\zeta ; u) \frac{z^{j}}{j !} .
\end{aligned}
$$

In view of Equation (51), we complete the proof.

Theorem 14. Let $j \in \mathbb{N}$ and $k \in \mathbb{Z}$. Then,

$$
\mathbb{H}_{j, \lambda, p}^{(k)}(\zeta ; u)=\sum_{s=0}^{j} \sum_{r=0}^{s}\left(\begin{array}{l}
j \\
s
\end{array}\right) \frac{p(r+1)(1)_{r+1, \lambda}(r+1) ! S_{1, \lambda}(r+1, s+1) \mathbb{H}_{j-s, \lambda}(u)(1-u)^{s}}{(r+1)^{k}(s+1)} .
$$

Proof. Using Equation (49), we have the following:

$$
\begin{aligned}
\sum_{j=0}^{\infty} & \mathbb{H}_{j, \lambda, p}^{(k)}(u) \frac{z^{j}}{j !}=\frac{u_{k, \lambda}\left(\log _{\lambda}(1+(1-u) z) \mid p\right)}{z\left(e_{\lambda}(z)-u\right)} \\
& =\frac{1}{z\left(e_{\lambda}(z)-u\right)} \sum_{r=1}^{\infty} \frac{p(r)(1)_{r, \lambda}}{r^{k}}\left(\log _{\lambda}(1+(1-u) z)\right)^{r} \\
& =\frac{1}{z\left(e_{\lambda}(z)-u\right)} \sum_{r=0}^{\infty} \frac{p(r+1)(1)_{r+1, \lambda}(r+1) !}{(r+1)^{k}} \sum_{l=r+1}^{\infty} S_{1, \lambda}(r+1, l)(1-u)^{l} \frac{z^{l}}{l !} \\
& =\left(\sum_{j=0}^{\infty} \mathbb{H}_{j, \lambda}(u) \frac{z^{j}}{j !}\right)\left(\sum_{r=0}^{\infty} \sum_{l=0}^{r} \frac{p(r+1)(1)_{r+1, \lambda}(r+1) !}{(r+1)^{k}} \frac{S_{1, \lambda}(r+1, l+1)}{l+1} \frac{z^{l}}{l !}\right) \\
& =\sum_{j=0}^{\infty}\left(\sum_{s=0}^{\infty} \sum_{r=0}^{s}\left(\begin{array}{l}
j \\
s
\end{array}\right) \frac{p(r+1)(1)_{r+1, \lambda}(r+1) ! S_{1, \lambda}(r+1, s+1) \mathbb{H}_{j-s, \lambda}(u)(1-u)^{s}}{(r+1)^{k}(s+1)}\right) \frac{z^{j}}{j !}
\end{aligned}
$$

Therefore, by Equations (49) and (53), we get the result.

Corollary 15. Let $j \geq 0$. Then, we have the following:

$$
\mathbb{H}_{j, \lambda, 1 / \Gamma}^{(k)}(u)=\mathbb{H}_{j, \lambda}^{(k)}(u)=\sum_{s=0}^{n} \sum_{r=0}^{s}\left(\begin{array}{l}
j \\
l
\end{array}\right) \frac{S_{1, \lambda}(r+1, s+1) \mathbb{H}_{j-s, \lambda}(u)}{(r+1)^{k-1}(s+1)}
$$

Theorem 16. Let $j \geq 0$. Then, we have the following:

$$
\mathbb{H}_{j, \lambda, p}^{(k)}(\zeta ; u)=\sum_{r=0}^{j} \sum_{s=0}^{r}\left(\begin{array}{l}
j \\
r
\end{array}\right) H_{j-r, \lambda, p}^{(k)}(u)(\zeta)_{(r)} S_{2, \lambda}(r, s)
$$

Proof. By Equation (49), we have the following:

$$
\begin{aligned}
\sum_{j=0}^{\infty} & H_{j, \lambda}^{(k, p)}(x ; u) \frac{z^{j}}{j !}=\frac{u_{k, \lambda}\left(\log _{\lambda}(1+(1-u) z) \mid p\right)}{z\left(e_{\lambda}(z)-u\right)}\left(e_{\lambda}(z)-1+1\right)^{\zeta} \\
= & \frac{u_{k, \lambda}\left(\log _{\lambda}(1+(1-u) z) \mid p\right)}{z\left(e_{\lambda}(z)-u\right)} \sum_{l=0}^{\infty}\left(\begin{array}{c}
\zeta \\
l
\end{array}\right)\left(e_{\lambda}(z)-1\right)^{l} \\
= & \left(\sum_{j=0}^{\infty} H_{j, \lambda, p}^{(k)}(u) \frac{z^{j}}{j !}\right)\left(\sum_{l=0}^{\infty}(\zeta)_{l} \sum_{m=l}^{\infty} S_{2, \lambda}(m, l) \frac{z^{m}}{m !}\right) \\
= & \sum_{j=0}^{\infty}\left(\sum_{r=0}^{j} \sum_{s=0}^{r}\left(\begin{array}{c}
n \\
r
\end{array}\right) H_{j-r, \lambda, p}^{(k)}(u)(\zeta)_{(r)} S_{2, \lambda}(r, s)\right) \frac{z^{j}}{j !} .
\end{aligned}
$$

By Equation (56), we obtain the result.

Theorem 17. Let $j \geq 0$. Then,

$$
\mathbb{H}_{j, \lambda, p}^{(k)}(\zeta ; u)=j \mathbb{H}_{j-1, \lambda, p}^{(k)}(\zeta ; u)
$$

Proof. When we consider Equation (49), we see that

$$
\Delta_{\lambda}\left(\sum_{j=0}^{\infty} \mathbb{H}_{n, \lambda, p}^{(k)}(\zeta ; u) \frac{z^{j}}{j !}\right)=\Delta_{\lambda}\left(\frac{u_{k, \lambda}\left(\log _{\lambda}(1+(1-u) z) \mid p\right)}{z\left(e_{\lambda}(z)-u\right)}(1+\lambda z)^{v / \lambda}\right)
$$

and then we have the following:

$$
\begin{aligned}
\sum_{j=0}^{\infty} & \Delta_{\lambda} \mathbb{H}_{j, \lambda, p}^{(k)}(\zeta ; u) \frac{z^{j}}{j !}=\frac{u_{k, \lambda}\left(\log _{\lambda}(1+(1-u) z)\right)}{z\left(e_{\lambda}(z)-u\right)} \Delta_{\lambda} e_{\lambda}^{\zeta}(z) \\
& =\frac{u_{k, \lambda}\left(\log _{\lambda}(1+(1-u) z)\right)}{z\left(e_{\lambda}(z)-u\right)} e_{\lambda}^{\zeta}(z) z \\
& =\sum_{j=0}^{\infty} \mathbb{H}_{j, \lambda, p}^{(k)}(\zeta ; u) \frac{z^{j+1}}{j !} .
\end{aligned}
$$

Therefore, by Equation (59), we complete the proof. 
Theorem 18. Let $j \geq 0$ and $k \in \mathbb{Z}$. Then, we have the following:

$$
\frac{\partial}{\partial \zeta} \mathbb{H}_{j, \lambda, p}^{(k)}(\zeta ; u)=\sum_{r=0}^{j}\left(\begin{array}{l}
j \\
r
\end{array}\right) \mathbb{H}_{j-r, \lambda, p}^{(k)}(\zeta ; u)(1)_{r, \lambda} .
$$

Proof. In Equation (49), we consider that

$$
\begin{aligned}
& \frac{\partial}{\partial \zeta}\left(\sum_{j=0}^{\infty} \mathbb{H}_{j, \lambda, p}^{(k)}(\zeta ; u) \frac{z^{j}}{j !}\right) \\
& \quad=\frac{\partial}{\partial \zeta}\left(\frac{u_{k, \lambda}\left(\log _{\lambda}(1+(1-u) z) \mid p\right)}{z\left(e_{\lambda}(z)-u\right)}(1+\lambda z)^{\zeta / \lambda}\right) \sum_{j=0}^{\infty} \frac{\partial}{\partial \zeta} \mathbb{H}_{j, \lambda, p}^{(k)}(\zeta ; u) \frac{z^{j}}{j !} \\
& \quad=\frac{u_{k, \lambda}\left(\log _{\lambda}(1+(1-u) z)\right)}{z\left(e_{\lambda}(z)-u\right)} \frac{\partial}{\partial \zeta}(1+\lambda z)^{\zeta / \lambda} \\
& \quad=\frac{u_{k, \lambda}\left(\log _{\lambda}(1+(1-u) z)\right)}{z\left(e_{\lambda}(z)-u\right)}(1+\lambda z)^{\zeta / \lambda}(1+\lambda z)^{1 / \lambda} \\
& \quad=\left(\sum_{j=0}^{\infty} \mathbb{H}_{j, \lambda, p}^{(k)}(\zeta ; u) \frac{z^{j}}{j !}\right)\left(\sum_{r=0}^{\infty}(1)_{r, \lambda} \frac{z^{r}}{r !}\right) \\
& \quad=\sum_{j=0}^{\infty}\left(\sum_{r=0}^{j}\left(\begin{array}{l}
j \\
r
\end{array}\right) \mathbb{H}_{j-r, \lambda, p}^{(k)}(\zeta ; u)(1)_{r, \lambda}\right) \frac{z^{j}}{j !} .
\end{aligned}
$$

By Equation (61), we complete the proof.

\section{Degenerate Poly-Frobenius-Euler Polynomials of Complex Variables}

In this section, we define the Frobenius-Euler polynomials of the complex variables. We consider the degenerate cosine function and degenerate sine function. Using the degenerate cosine function and the degenerate sine function, we introduce the degenerate cosine poly-Frobenius-Euler polynomials and degenerate sine poly-Frobenius-Euler polynomials.

In [5], Kim et al. defined the degenerate $\operatorname{sine} \sin _{\lambda} z$ and cosine $\cos _{\lambda} z$ functions by the following:

$$
\begin{aligned}
& \sin _{\lambda}^{(\zeta)}(z)=\frac{e_{\lambda}^{i x}(z)-e_{\lambda}^{-i \zeta}(z)}{2 i}, \\
& \cos _{\lambda}^{(\zeta)}(z)=\frac{e_{\lambda}^{i \zeta}(z)+e_{\lambda}^{-i \zeta}(z)}{2},
\end{aligned}
$$

where $i=\sqrt{-1}$. Note that $\lim _{\lambda \longrightarrow 0} \sin _{\lambda}^{(\zeta)}(z)=\sin \zeta z$ and $\lim _{\lambda \longrightarrow 0} \cos _{\lambda}^{(\zeta)}(z)=\cos \zeta z$. From Equation (62), it is readily seen that

$$
e_{\lambda}^{i \zeta}(z)=\cos _{\lambda}^{(\zeta)}(z)+i \sin _{\lambda}^{(\zeta)}(z)
$$

By these functions in Equation (62), the degenerate sinepolynomials $S_{j, \lambda}(\zeta, \eta)$ and degenerate cosine-polynomials $C_{j, \lambda}(\zeta, \eta)$ are introduced by Kim et al. [5] as follows:

$$
\sum_{j=0}^{\infty} S_{j, \lambda}(\zeta, \eta) \frac{z^{j}}{j !}=e_{\lambda}^{\zeta}(z) \sin _{\lambda}^{(\eta)}(z)
$$

$$
\sum_{j=0}^{\infty} C_{j, \lambda}(\zeta, \eta) \frac{z^{j}}{j !}=e_{\lambda}^{\zeta}(z) \cos _{\lambda}^{(\eta)}(z)
$$

Several properties of these polynomials in Equations (64) and (65) were studied and investigated by Kim et al. [5]. Also, by means of these functions, Kim et al. [5] introduced the degenerate Euler and Bernoulli polynomials of complex variable and investigate some of their properties. Motivated and inspired by these considerations above, we define degenerate poly-Frobenius-Euler polynomials of complex variable as follows:

$$
\begin{aligned}
& \sum_{j=0}^{\infty} \mathbb{H}_{j, \lambda}^{(k)}(\zeta+i \eta ; u) \frac{z^{j}}{j !}=\frac{E i_{k, \lambda}\left(\log _{\lambda}(1+(1-u) z)\right)}{z\left(e_{\lambda}(z)-u\right)} e_{\lambda}^{(\zeta+i \eta)}(z) \\
& =\frac{E i_{k, \lambda}\left(\log _{\lambda}(1+(1-u) z)\right)}{z\left(e_{\lambda}(z)-u\right)} e_{\lambda}^{\zeta}(z)\left[\cos _{\lambda}^{(\eta)}(z)+i \sin _{\lambda}^{(\eta)}(z)\right], \\
& \sum_{j=0}^{\infty} \mathbb{H}_{j, \lambda}^{(k)}(\zeta-i \eta ; u) \frac{z^{j}}{j !}=\frac{E i_{k, \lambda}\left(\log _{\lambda}(1+(1-u) z)\right)}{z\left(e_{\lambda}(z)-u\right)} e_{\lambda}^{(\zeta-i \eta)}(z) \\
& =\frac{E i_{k, \lambda}\left(\log _{\lambda}(1+(1-u) z)\right)}{z\left(e_{\lambda}(z)-u\right)} e_{\lambda}^{\zeta}(z)\left[\cos _{\lambda}^{(\eta)}(z)-i \sin _{\lambda}^{(\eta)}(z)\right] .
\end{aligned}
$$

From Equations (66) and (67), we get the following:

$$
\begin{aligned}
& \frac{E i_{k, \lambda}\left(\log _{\lambda}(1+(1-u) z)\right)}{z\left(e_{\lambda}(z)-u\right)} e_{\lambda}^{\zeta}(z) \cos _{\lambda}^{(\eta)}(z) \\
& =\sum_{j=0}^{\infty} \frac{\mathbb{H}_{j, \lambda}^{(k)}(\zeta+i \eta ; u)+\mathbb{H}_{j, \lambda}^{(k)}(\zeta-i \eta ; u)}{2} \frac{z^{j}}{j !}, \\
& \frac{E i_{k, \lambda}\left(\log _{\lambda}(1+(1-u) z)\right)}{z\left(e_{\lambda}(z)-u\right)} e_{\lambda}^{\zeta}(z) \sin _{\lambda}^{(\eta)}(z) \\
& =\sum_{j=0}^{\infty} \frac{\mathbb{H}_{j, \lambda}^{(k)}(\zeta+i \eta ; u)-\mathbb{H}_{j, \lambda}^{(k)}(\zeta-i \eta ; u)}{2 i} \frac{z^{j}}{j !} .
\end{aligned}
$$

Now, we define the degenerate cosine poly-FrobeniusEuler polynomials and the degenerate sine poly-FrobeniusEuler polynomials, respectively, as follows:

$$
\sum_{j=0}^{\infty} \mathbb{H}_{j, \lambda}^{[k, c]}(\zeta, \eta ; u) \frac{z^{j}}{j !}=\frac{E i_{k, \lambda}\left(\log _{\lambda}(1+(1-u) z)\right)}{z\left(e_{\lambda}(z)-u\right)} e_{\lambda}^{\zeta}(z) \cos _{\lambda}^{(\eta)}(z),
$$

$$
\sum_{j=0}^{\infty} \mathbb{H}_{j, \lambda}^{[k, s]}(\zeta, \eta ; u) \frac{z^{j}}{j !}=\frac{E i_{k, \lambda}\left(\log _{\lambda}(1+(1-u) z)\right)}{z\left(e_{\lambda}(z)-u\right)} e_{\lambda}^{\zeta}(z) \sin _{\lambda}^{(\eta)}(z) .
$$


Theorem 19. The following results hold true:

$$
\mathbb{H}_{j, \lambda}^{[k, c]}(\zeta, \eta ; u)=\frac{1}{2} \sum_{r=0}^{j}\left(\begin{array}{l}
j \\
r
\end{array}\right) \mathbb{H}_{j-r, \lambda}^{[k, c]}(\zeta, \eta ; u) \sum_{l=0}^{r}\left(\begin{array}{l}
r \\
l
\end{array}\right)(\zeta)_{r-l, \lambda}\left((i \eta)_{l, \lambda}+(-i \eta)_{l, \lambda}\right),
$$

$\mathbb{H}_{j, \lambda}^{[k, s]}(\zeta, \eta ; u)=\frac{1}{2 i} \sum_{r=0}^{j}\left(\begin{array}{l}j \\ r\end{array}\right) \mathbb{H}_{j-r, \lambda}^{[k, s]}(\zeta, \eta ; u) \sum_{l=0}^{r}\left(\begin{array}{l}r \\ l\end{array}\right)(\zeta)_{r-l, \lambda}\left((i \eta)_{l, \lambda}-(-i \eta)_{l, \lambda}\right)$.

Proof. From Equation (69), we have the following:

$$
\begin{aligned}
\sum_{j=0}^{\infty} & \mathbb{H}_{j, \lambda}^{[k, c]}(\zeta, \eta ; u) \frac{z^{j}}{j !}=\frac{E i_{k, \lambda}\left(\log _{\lambda}(1+(1-u) z)\right)}{z\left(e_{\lambda}(z)-u\right)} e_{\lambda}^{\zeta}(z) \cos _{\lambda}^{(\eta)}(z) \\
& =\frac{E i_{k, \lambda}\left(\log _{\lambda}(1+(1-u) z)\right)}{z\left(e_{\lambda}(z)-u\right)} \frac{1}{2} \sum_{r=0}^{\infty} \sum_{l=0}^{r}\left(\begin{array}{l}
r \\
l
\end{array}\right)(\zeta)_{r-l, \lambda}\left((i \eta)_{l, \lambda}+(-i \eta)_{l, \lambda}\right) \frac{z^{r}}{r !} \\
& =\sum_{j=0}^{\infty}\left(\frac{1}{2} \sum_{r=0}^{j}\left(\begin{array}{l}
j \\
r
\end{array}\right) \mathbb{H}_{j-r, \lambda}^{[k, c]}(\zeta, \eta ; u) \sum_{l=0}^{r}\left(\begin{array}{l}
r \\
l
\end{array}\right)(\zeta)_{r-l, \lambda}\left((i \eta)_{l, \lambda}+(-i \eta)_{l, \lambda}\right)\right) \frac{z^{j}}{j !}
\end{aligned}
$$

By Equation (73), we get Equation (71). Similarly, by using Equations (70) and (3), we can easily find the result (Equation (72)).

Theorem 20. The following results hold true:

$$
\begin{aligned}
& \mathbb{H}_{j, \lambda}^{[k, c]}\left(\zeta_{1}+\zeta_{2} ; u\right)=\sum_{m=0}^{j}\left(\begin{array}{c}
j \\
m
\end{array}\right) \sum_{r=0}^{m} S_{2, \lambda}^{\left(\zeta_{1}\right)}(m, r)\left(\zeta_{2}\right)_{r} \mathbb{H}_{j, \lambda}^{[k, c]}(0, \eta ; u), \\
& \mathbb{H}_{j, \lambda}^{[k, s]}\left(\zeta_{1}+\zeta_{2} ; u\right)=\sum_{m=0}^{j}\left(\begin{array}{c}
j \\
m
\end{array}\right) \sum_{r=0}^{m} S_{2, \lambda}^{\left(\zeta_{1}\right)}(m, r)\left(\zeta_{2}\right)_{r} \mathbb{H}_{j, \lambda}^{[k, s]}(0, \eta ; u) .
\end{aligned}
$$

Proof. From Equations (3) and (69), we have the following:

$$
\begin{aligned}
\sum_{j=0}^{\infty} & \mathbb{H}_{j, \lambda}^{[k, c]}\left(\zeta_{1}+\zeta_{2} ; u\right) \frac{z^{j}}{j !}=\frac{e_{\lambda}^{\left(\zeta_{1}+\zeta_{2}\right)}(z) E i_{k, \lambda}\left(\log _{\lambda}(1+(1-u) z)\right)}{z\left(e_{\lambda}(z)-u\right)} \cos _{\lambda}^{(\eta)}(z) \\
& =\sum_{m=0}^{\infty} \sum_{r=0}^{m} S_{2, \lambda}^{\left(\zeta_{1}\right)}(m, r)\left(\zeta_{2}\right)_{r} \frac{z^{m}}{m !} \sum_{j=0}^{\infty} \mathbb{H}_{j, \lambda}^{[k, c]}(0, \eta ; u) \frac{z^{j}}{j !} \\
& =\sum_{j=0}^{\infty}\left(\sum_{m=0}^{j}\left(\begin{array}{c}
j \\
m
\end{array}\right) \sum_{r=0}^{m} S_{2, \lambda}^{\left(\zeta_{1}\right)}(m, r)\left(\zeta_{2}\right)_{r} \mathbb{H}_{j, \lambda}^{[k, c]}(0, \eta ; u)\right) \frac{z^{j}}{j !} .
\end{aligned}
$$

In view of Equation (76), we get Equation (74). Similarly, by using Equations (3) and (70), we require at the desired result (Equation (75)).
Theorem 21. The following results hold true:

$$
\begin{aligned}
\mathbb{H}_{j, \lambda}^{[k, c]}\left(\zeta_{1}+\zeta_{2}, \eta_{1}+\eta_{2} ; u\right)=\sum_{r=0}^{j}\left(\begin{array}{c}
j \\
r
\end{array}\right) \\
\cdot\left[\mathbb{H}_{j-r, \lambda}^{[k, c]}\left(\zeta_{1}, \eta_{1} ; u\right) C_{r, \lambda}\left(\zeta_{2}, \eta_{2}\right)-\mathbb{H}_{j, \lambda}^{[k, s]}\left(\zeta_{1}, \eta_{1} ; u\right) S_{r, \lambda}\left(\zeta_{2}, \eta_{2}\right)\right],
\end{aligned}
$$

$$
\begin{aligned}
& \mathbb{H}_{j, \lambda}^{[k, s]}\left(\zeta_{1}+\zeta_{2}, \eta_{1}+\eta_{2} ; u\right)=\sum_{r=0}^{j}\left(\begin{array}{c}
j \\
r
\end{array}\right) \\
& \cdot\left[\mathbb{H}_{j-r, \lambda}^{[k, s]}\left(\zeta_{1}, \eta_{1} ; u\right) C_{r, \lambda}\left(\zeta_{2}, \eta_{2}\right)-\mathbb{H}_{j, \lambda}^{[k, c]}\left(\zeta_{1}, \eta_{1} ; u\right) S_{m, \lambda}\left(\zeta_{2}, \eta_{2}\right)\right] .
\end{aligned}
$$

Proof. From Equation (69), we have the following:

$$
\begin{aligned}
\sum_{j=0}^{\infty} \mathbb{H}_{j, \lambda}^{[k, c]}\left(\zeta_{1}+\zeta_{2}, \eta_{1}+\eta_{2} ; u\right) \frac{z^{j}}{j !}=\frac{e_{\lambda}^{\left(\zeta_{1}+\zeta_{2}\right)}(z) E i_{k, \lambda}\left(\log _{\lambda}(1+(1-u) z)\right)}{z\left(e_{\lambda}(z)-u\right)} & \cdot\left[\cos _{\lambda}^{\left(\eta_{1}\right)}(z) \cos _{\lambda}^{\left(\eta_{2}\right)}(z)-\sin _{\lambda}^{\left(\eta_{1}\right)}(z) \sin _{\lambda}^{\left(\eta_{2}\right)}(z)\right] \\
= & \sum_{j=0}^{\infty} \mathbb{H}_{j, \lambda}^{[k, c]}\left(\zeta_{1}, \eta_{1} ; u\right) \frac{z^{j}}{j !} \sum_{r=0}^{\infty} C_{r, \lambda}\left(\zeta_{2}, \eta_{2}\right) \frac{z^{r}}{r !} \\
& -\sum_{j=0}^{\infty} \mathbb{H}_{j, \lambda}^{[k, s]}\left(\zeta_{1}, \eta_{1} ; u\right) \frac{z^{j}}{j !} \sum_{r=0}^{\infty} S_{r, \lambda}\left(\zeta_{2}, \eta_{2}\right) \frac{z^{r}}{r !} \\
= & \sum_{j=0}^{\infty}\left(\sum_{r=0}^{j}\left(\begin{array}{l}
j \\
r
\end{array}\right) \mathbb{H}_{j-r, \lambda}^{[k, c]}\left(\zeta_{1}, \eta_{1} ; u\right) C_{r, \lambda}\left(\zeta_{2}, \eta_{2}\right)\right. \\
& \left.-\mathbb{H}_{j, \lambda}^{[k, s]}\left(\zeta_{1}, \eta_{1} ; u\right) S_{m, \lambda}\left(\zeta_{2}, \eta_{2}\right)\right) \frac{z^{j}}{j !} .
\end{aligned}
$$

Comparing the coefficients of $z$ on both sides, we get Equation (77). Similarly, by using Equation (70), we can easily get Equation (78).

Corollary 22. On setting $\zeta_{1}=\zeta_{2}=\zeta$ and $\eta_{1}=\eta_{2}=\eta$ in Theorem 16, we have the following:

$$
\begin{aligned}
& \mathbb{H}_{j, \lambda}^{[k, c]}(2 \zeta, 2 \eta ; u)=\sum_{r=0}^{j}\left(\begin{array}{l}
j \\
r
\end{array}\right)\left[\mathbb{H}_{j-r, \lambda}^{[k, c]}(\zeta, \eta ; u) C_{r, \lambda}(\zeta, \eta)-\mathbb{H}_{j, \lambda}^{[k, s]}(\zeta, \eta ; u) S_{r, \lambda}(\zeta, \eta)\right], \\
& \mathbb{H}_{j, \lambda}^{[k, s]}(2 \zeta, 2 \eta ; u)=\sum_{r=0}^{j}\left(\begin{array}{l}
j \\
r
\end{array}\right)\left[\mathbb{H}_{j-r, \lambda}^{[k, s]}(\zeta, \eta ; u) C_{r, \lambda}(\zeta, \eta)-\mathbb{H}_{j, \lambda}^{[k, c]}(\zeta, \eta ; u) S_{m, \lambda}(\zeta, \eta)\right] .
\end{aligned}
$$

\section{Conclusions}

In this paper, we defined the degenerate poly-FrobeniusEuler polynomials by employing the modified degenerate polyexponential functions. We have established some identities and relations between degenerate Whitney numbers and degenerate Stirling numbers of the first kind. Also, we have established addition formulas and derivative formulas of degenerate poly-Frobenius-Euler polynomials. In the last 
section, we have defined degenerate poly-Frobenius-Euler polynomials of complex variables and then we have derived several properties and relations.

\section{Data Availability}

No data were used to support the study.

\section{Conflicts of Interest}

The authors declare no conflict of interest.

\section{Authors' Contributions}

All authors contributed equally to the manuscript and typed, read, and approved the final manuscript.

\section{Acknowledgments}

This work was supported by the Taif University Researchers Supporting Project (TURSP-2020/246), Taif University, Taif, Saudi Arabia.

\section{References}

[1] L. Carlitz, "Degenerate Stirling," Bernoulli and Eulerian numbers. Utilitas Math., vol. 15, pp. 51-88, 1979.

[2] L. Carlitz, "A degenerate Staudt-Clausen theorem," Archiv der Mathematik, vol. 7, no. 1, pp. 28-33, 1956.

[3] D. S. Kim and T. Kim, "A note on polyexponential and unipoly functions," Russian Journal of Mathematical Physics, vol. 26, no. 1, pp. 40-49, 2019.

[4] D. S. Kim and T. Kim, "A note on a new type of degenerate Bernoulli numbers," Russian Journal of Mathematical Physics, vol. 27, no. 2, pp. 227-235, 2020.

[5] D. S. Kim, T. Kim, and H. Lee, "A note on degenerate Euler and Bernoulli polynomials of complex variable," Symmetry., vol. 11, no. 9, p. 1168, 2019.

[6] T. Kim, H. I. Kwon, and J. J. Seo, "On the degenerate Frobenius-Euler polynomials,” July 2015, http://arxiv.org/ abs/1507.04846v1.

[7] D. S. Kim, T. Kim, and J. J. Seo, "Higher-order Bernoulli, Frobenius-Euler and Euler polynomials," Journal of Computational Analysis and Applications, vol. 17, no. 1, pp. 147-155, 2014.

[8] T. Kim, D. S. Kim, H.-Y. Kim, and J. Kwon, "Some results on degenerate Daehee and Bernoulli numbers and polynomials," Advances in Difference Equations, vol. 311, 13 pages, 2020.

[9] T. Kim, D. S. Kim, J. Kwon, and H. Y. Kim, "A note on degenerate Genocchi and poly-Genocchi numbers and polynomials," Journal of Inequalities and Applications, vol. 2020, no. $1,2020$.

[10] G. Muhiuddin, W. A. Khan, and U. Duran, "Two variable type 2 Fubini polynomials," Mathematics., vol. 9, no. 281, pp. 1-13, 2021.

[11] G. Muhiuddin, W. A. Khan, U. Duran, and D. al-Kadi, "Some identities of the degenerate multi-poly-Bernoulli polynomials of complex variable," Journal of Function Spaces, vol. 2021, Article ID 7172054, 8 pages, 2021.

[12] G. Muhiuddin, W. A. Khan, U. Duran, and D. Al-Kadi, “A new class of higher-order hypergeometric Bernoulli polynomials associated with Lagrange-Hermite polynomials," Symmetry., vol. 13, no. 4, p. 648, 2021.

[13] S. K. Sharma, W. A. Khan, and C. S. Ryoo, "A parametric kind of the degenerate Fubini numbers and polynomials," Mathematics., vol. 8, no. 3, p. 405, 2020.

[14] S. Kumar Sharma, W. A. Khan, S. Araci, and S. S. Ahmed, "New type of degenerate Daehee polynomials of the second kind," Advances in Difference Equations, vol. 2020, no. 1, 2020.

[15] S. K. Sharma, W. A. Khan, S. Araci, and S. S. Ahmed, "New construction of type 2 degenerate central Fubini polynomials with their certain properties," Advances in Difference Equations, vol. 2020, no. 1, 2020.

[16] B. Kurt, "Poly-Frobenius-Euler polynomials," Proceedings of the International Conferences on Numerical Analysis and Applied Mathematics. AIP Conference Proceedings, vol. 1863, p. 300019-1-300019-3, 2016.

[17] B. Kurt and Y. Simsek, "On the generalized Apostol-type Frobenius-Euler polynomials," Adv. Difference Equ., vol. 2013, no. 1, pp. 1-9, 2013.

[18] T. Kim, "A note on degenerate Stirling polynomials of the second kind," Proceedings of the Jangjeon Mathematical Society, vol. 20, no. 3, pp. 319-331, 2017.

[19] D. V. Dolgy and W. A. Khan, "A note on type-two degenerate poly-Changhee polynomials of the second kind," Symmetry., vol. 13, no. 4, p. 579, 2021.

[20] M. Kaneko, "Poly-Bernoulli numbers," Journal de Théorie des Nombres de Bordeaux, vol. 9, no. 1, pp. 221-228, 1997. 